PROCEEDINGS OF THE

AMERICAN MATHEMATICAL SOCIETY

Volume 133, Number 5, Pages 1475-1484

S 0002-9939(04)07679-8

Article electronically published on November 19, 2004

\title{
A VECTORIAL INVERSE NODAL PROBLEM
}

\author{
YAN-HSIOU CHENG, CHUNG-TSUN SHIEH, AND C. K. LAW
}

(Communicated by Carmen C. Chicone)

Abstract. Consider the vectorial Sturm-Liouville problem:

$$
\left\{\begin{array}{l}
-\mathbf{y}^{\prime \prime}(x)+P(x) \mathbf{y}(x)=\lambda I_{d} \mathbf{y}(x) \\
A \mathbf{y}(0)+I_{d} \mathbf{y}^{\prime}(0)=\mathbf{0} \\
B \mathbf{y}(1)+I_{d} \mathbf{y}^{\prime}(1)=\mathbf{0}
\end{array}\right.
$$

where $P(x)=\left[p_{i j}(x)\right]_{i, j=1}^{d}$ is a continuous symmetric matrix-valued function defined on [0,1], and $A$ and $B$ are $d \times d$ real symmetric matrices. An eigenfunction $\mathbf{y}(x)$ of the above problem is said to be of type (CZ) if any isolated zero of its component is a nodal point of $\mathbf{y}(x)$. We show that when $d=2$, there are infinitely many eigenfunctions of type $(\mathrm{CZ})$ if and only if $(P(x), A, B)$ are simultaneously diagonalizable. This indicates that $(P(x), A, B)$ can be reconstructed when all except a finite number of eigenfunctions are of type (CZ). The results supplement a theorem proved by Shen-Shieh (the second author) for Dirichlet boundary conditions. The proof depends on an eigenvalue estimate, which seems to be of independent interest.

\section{INTRODUCTION}

The inverse nodal problem, first studied by J. McLaughlin ( 8 , see also 3 and [9]), is the problem of finding the potential function and the boundary conditions using only the set of nodal points of the eigenfunctions. Consider the scalar inverse nodal problem,

$$
\left\{\begin{array}{l}
-y^{\prime \prime}+q(x) y=\lambda y \\
y(0) \cos \alpha+y^{\prime}(0) \sin \alpha=0 \\
y(1) \cos \beta+y^{\prime}(1) \sin \beta=0
\end{array}\right.
$$

where $q \in L^{1}(0,1)$ and $\alpha, \beta \in[0, \pi)$. We also let $\lambda_{n}$ be the eigenvalue, $y_{n}$ the corresponding eigenfunction and $\left\{x_{k}^{(n)}: k=1,2, \ldots, n-1\right\}$ the nodal set of $y_{n}$ in $(0,1)$. It is now known that $(q, \alpha, \beta)$ can be uniquely determined by the nodal set up to a constant $(8,13])$, and can also be reconstructed from the nodal set ([3, 7, 13]).

Theorem 1.1 ([13, 7]). For almost every $x \in(0,1)$, with

$$
j=j_{n}(x)=\max \left\{i: x_{i}^{(n)} \leq x\right\}
$$

Received by the editors August 27, 2003 and, in revised form, February 4, 2004.

2000 Mathematics Subject Classification. Primary 34B24, 34C10.

(C)2004 American Mathematical Society Reverts to public domain 28 years from publication 
we have

$$
q(x)=\lim _{n \rightarrow \infty} 2 \lambda_{n}\left(\frac{\sqrt{\lambda_{n}} l_{j}^{(n)}}{\pi}-1\right)
$$

where $l_{i}^{(n)}=x_{i+1}^{(n)}-x_{i}^{(n)}$ is the nodal length. Furthermore, the boundary phases $\alpha$ and $\beta$ can also be reconstructed from the nodal data.

Note that in the above, if $\alpha$ and $\beta$ are known, then an asymptotic expansion of $\sqrt{\lambda_{n}}$ up to $O\left(n^{-2}\right)$ guarantees that $q$ can be approximated by the nodal data alone up to a constant. In fact, the convergence holds in $L^{1}(0,1)([4)$.

But so far, not much is known for the inverse nodal problem of the vectorial Sturm-Liouville system, although some work has been done on the corresponding inverse eigenvalue problems [10, 11]. Consider

$$
-\mathbf{y}^{\prime \prime}(x)+P(x) \mathbf{y}(x)=\lambda I_{d} \mathbf{y}(x),
$$

with boundary conditions

$$
\left\{\begin{array}{l}
A_{1} \mathbf{y}(0)+A_{2} \mathbf{y}^{\prime}(0)=\mathbf{0} \\
B_{1} \mathbf{y}(1)+B_{2} \mathbf{y}^{\prime}(1)=\mathbf{0}
\end{array}\right.
$$

where $P(x)=\left[p_{i j}(x)\right]_{i, j=1}^{d}$ is a continuous symmetric matrix-valued function defined on [0,1], $A_{i}, B_{i}, i=1,2$, are $d \times d$ matrices, and $I_{d}$ is the $d \times d$ identity matrix. If $A_{2}=B_{2}=0$, and $A_{1}, B_{1}$ are invertible, then (3) represents the Dirichlet boundary condition. If $A_{1}=B_{1}=0$, and $A_{2}, B_{2}$ are invertible, then (3) represents the Neumann boundary condition.

In order that the system be self-adjoint, we require the boundary matrices to satisfy

$$
\begin{aligned}
& A_{1} A_{2}^{*}=A_{2} A_{1}^{*}, \quad B_{1} B_{2}^{*}=B_{2} B_{1}^{*}, \\
& \operatorname{rank}\left[A_{1}, A_{2}\right]=\operatorname{rank}\left[B_{1}, B_{2}\right]=d
\end{aligned}
$$

([5]). In short, vectorial systems satisfying (40) can be transformed to a first-order Dirac system, which is self-adjoint because of (4a) ([6]). Note that if $A_{2}$ and $B_{2}$ are invertible, then (4) reduces to

$$
A_{2}^{-1} A_{1}=\left(A_{2}^{-1} A_{1}\right)^{*}, \quad B_{2}^{-1} B_{1}=\left(B_{2}^{-1} B_{1}\right)^{*} .
$$

Thus the real matrices $A_{2}^{-1} A_{1}$ and $B_{2}^{-1} B_{1}$ are symmetric. In this case, the boundary condition (3) can be reduced to a simpler one:

$$
\begin{aligned}
& A y(0)+I_{d} y^{\prime}(0)=0, \\
& B y(1)+I_{d} y^{\prime}(1)=0
\end{aligned}
$$

where $A, B$ are symmetric and $I_{d}$ is the $d \times d$ identity matrix. We shall concentrate on the study of the vectorial system with this rather general boundary condition, which includes the Neumann boundary condition.

Let $\mathbf{y}(x)$ be a continuous $d$-dimensional vector-valued function defined on $[0,1]$. A point $x_{0} \in[0,1]$ is called a nodal point of $\mathbf{y}(x)$ if $\mathbf{y}\left(x_{0}\right)=\mathbf{0}$. We say that $\mathbf{y}(x)$ is a vector-valued function of type (CZ) (or $\mathbf{y}$ has common zero property) if all the isolated zeros of its components are nodal points. $P(x)$ is called simultaneously diagonalizable if there is a unitary matrix $Q$ such that $Q^{*} P(x) Q$ is a diagonal matrix-valued function. A vectorial Sturm-Liouville problem is said to be ( $\star$ )-simultaneously diagonalizable if $P(x), A_{i}, B_{i}(i=1,2)$ are all simultaneously 
diagonalizable by the same matrix, i.e. $Q^{*} P(x) Q, Q^{*} A_{i} Q$ and $Q^{*} B_{i} Q(i=1,2)$ are diagonal.

If there is a unitary matrix $Q$ such that $Q^{*} P Q, Q^{*} A_{i} Q, Q^{*} B_{i} Q(i=1,2)$ are all diagonal, then (2) and (3) can be reduced to $d$ one-dimensional systems, and each system has infinitely many eigenfunctions that induce the eigenfunctions of (2) and (3) of type (CZ). Thus we have the following lemma about the nodal property of the vectorial Sturm-Liouville problem.

Lemma 1.2. If the vectorial Sturm-Liouville problem (2) and (3) is ( $\star$ )-simultaneously diagonalizable, then there are infinitely many eigenfunctions that are of type $(C Z)$.

The above nodal property for the vectorial Sturm-Liouville problem motivates the whole study. In 1999, Shen and Shieh proved the converse of Lemma 1.2 for the Dirichlet problem when dimension $d=2$. It seems to be the first vectorial inverse nodal problem studied.

Theorem 1.3 (12, Theorem 2.6]). Let $d=2$. Suppose the Dirichlet vectorial Sturm-Liouville problem (2) has infinitely many eigenfunctions $\left\{\mathbf{y}_{n_{j}}(x)\right\}_{j=1}^{\infty}$, which are of type $(C Z)$. Then $P(x)$ is simultaneously diagonalizable.

It would be interesting to see if the above result also holds for arbitrary boundary conditions. Recall that in the scalar case, it was shown in [7] that the smoothness of the potential function depends only on the nodal data, regardless of the boundary conditions. In the present case, the answer is also affirmative for the general boundary condition (5). In fact, the result is more complete. Below is our main theorem.

Theorem 1.4. Let $d=2$. Suppose the vectorial Sturm-Liouville problem (2) with boundary conditions (5) has infinitely many eigenfunctions $\left\{\mathbf{y}_{n_{j}}(x)\right\}_{j=1}^{\infty}$, which are of type (CZ). Then the problem is ( $\star$ )-simultaneously diagonalizable.

Of course, if more is known about the eigenfunctions $\left\{\mathbf{y}_{n_{j}}(x)\right\}_{j=1}^{\infty}$, we can obtain more information about $(P(x), A, B)$. Then we have the following corollary.

Corollary 1.5. If $d=2$ and all except a finite number of eigenfunctions of the vectorial problem (2) and (5) are of type (CZ), then the problem can be uniquely determined up to a unitary matrix and can be reconstructed.

Proof. By Theorem 1.4, we see that $P(x), A$ and $B$ are (simultaneously) similar to some $Q^{*} P Q \operatorname{diag}\left\{u_{1}(x), u_{2}(x)\right\}, Q^{*} A Q=\operatorname{diag}\left\{a_{1}, a_{2}\right\}$ and $Q^{*} B Q=\operatorname{diag}\left\{b_{1}, b_{2}\right\}$, respectively. Thus we obtain two scalar Sturm-Liouville systems by the transformation $Q^{*} \mathbf{y}=[v, w]^{T}$ :

$$
\left\{\begin{array}{l}
-v^{\prime \prime}(x)+u_{1}(x) v(x)=\mu v(x) \\
a_{1} v(0)+v^{\prime}(0)=0 \\
b_{1} v(1)+v^{\prime}(1)=0
\end{array}\right.
$$

and

$$
\left\{\begin{array}{l}
-w^{\prime \prime}(x)+u_{2}(x) w(x)=\rho w(x) \\
a_{2} w(0)+w^{\prime}(0)=0 \\
b_{2} w(1)+w^{\prime}(1)=0
\end{array}\right.
$$


From the assumption of the eigenfunctions, we can obtain two sets of nodal points, one for (6), and another for (7). Hence we can apply Theorem 1.1 to show that $\left(u_{1}(x), a_{1}, b_{1}\right)$ and $\left(u_{2}(x), a_{2}, b_{2}\right)$ can be reconstructed and so are uniquely determined up to a constant.

Our proof of Theorem 1.4 is based on a simplified proof of Theorem 1.3. Here, the eigenvalue asymptotics plays an important role. This eigenvalue estimate seems to be of independent interest. Carlson ([2]) used perturbation of operators to prove the estimate for another set of rather general boundary conditions, including the Dirichlet and Neumann boundary conditions, but our estimate is a lot simpler. We shall state and prove the eigenvalue estimate in section 2. Theorem 1.4 will be proved in section 3. For the sake of completeness, proofs of Lemma 1.2 and Lemma 3.1 are given in the appendices.

\section{Eigenvalue asymptotics}

In this section, we will study the asymptotic estimates of the eigenvalues and the nodal points. Results here are valid for arbitrary dimension $d \geq 2$. Consider the vectorial Sturm-Liouville equation (2) with boundary conditions (5)).

Lemma 2.1 ([1]). Let $Y(x, \lambda)$ denote the solution of the matrix differential equation

$$
\left\{\begin{array}{l}
-Y^{\prime \prime}+P(x) Y=\lambda I_{d} Y \\
Y(0, \lambda)=I_{d}, Y^{\prime}(0, \lambda)=-A
\end{array}\right.
$$

Then $Y(x, \lambda)$ satisfies

$$
Y(x, \lambda)=\cos (\sqrt{\lambda} x) I_{d}-\frac{\sin (\sqrt{\lambda} x)}{\sqrt{\lambda}} A+\int_{0}^{x} \frac{\sin (\sqrt{\lambda}(x-t))}{\sqrt{\lambda}} P(t) Y(t, \lambda) d t .
$$

Theorem 2.2. Let $\lambda_{n}$ be the $n$-th eigenvalue of the vectorial problem (2) and (5). Then let $m=\left[\frac{n}{d}\right]$, the floor function of $\frac{n}{d}$,

$$
\begin{aligned}
\sqrt{\lambda_{d m+j}} & =(m+1) \pi+\frac{a_{j}}{(m+1) \pi}+O\left(\frac{1}{m^{2}}\right) \\
& =(m+1) \pi+O\left(\frac{1}{m}\right), 1 \leq j \leq d,
\end{aligned}
$$

for sufficiently large $m$, where $a_{1} \leq a_{2} \leq \cdots \leq a_{d}$ are the eigenvalues of $B-A+$ $\frac{1}{2} \int_{0}^{1} P(t) d t$.

Proof. Suppose $Y(x, \lambda)$ is a solution of (8). Note that $|\sin (\sqrt{\lambda} x)| \leq \exp (|\operatorname{Im} \sqrt{\lambda}| x)$ and $|\cos (\sqrt{\lambda} x)| \leq \exp (|\operatorname{Im} \sqrt{\lambda}| x)$. Since $P(x) \in C\left([0,1], \mathbb{R}^{d}\right)$, we have

$$
\begin{aligned}
Y(x, \lambda) & =\cos (\sqrt{\lambda} x) I_{d}-\frac{\sin (\sqrt{\lambda} x)}{\sqrt{\lambda}} A+\int_{0}^{x} \frac{\sin (\sqrt{\lambda}(x-t))}{\sqrt{\lambda}} P(t) Y(t, \lambda) d t \\
& =\cos (\sqrt{\lambda} x) I_{d}+O\left(\frac{\exp |I m \sqrt{\lambda}|}{\sqrt{\lambda}}\right) .
\end{aligned}
$$


Also, since

$$
\begin{aligned}
& \int_{0}^{x} \cos (\sqrt{\lambda}(x-t)) P(t) Y(t, \lambda) d t \\
& =\int_{0}^{x}(\cos (\sqrt{\lambda} x) \cos (\sqrt{\lambda} t)+\sin (\sqrt{\lambda} x) \sin (\sqrt{\lambda} t)) \\
& \quad P(t)\left(\cos (\sqrt{\lambda} t) I_{d}+O\left(\frac{\exp |\operatorname{Im} \sqrt{\lambda}|}{\sqrt{\lambda}}\right)\right) d t \\
& =\cos (\sqrt{\lambda} x) \int_{0}^{x} \frac{1+\cos (2 \sqrt{\lambda} t)}{2} P(t) d t \\
& +\frac{\sin (\sqrt{\lambda} x)}{2} \int_{0}^{x} \sin (2 \sqrt{\lambda} t) P(t) d t+O\left(\frac{\exp 2|\operatorname{Im} \sqrt{\lambda}|}{\sqrt{\lambda}}\right) \\
& =\frac{\cos (\sqrt{\lambda} x)}{2} \int_{0}^{x} P(t) d t+o(\exp |\operatorname{Im} \sqrt{\lambda}|),
\end{aligned}
$$

we have

$$
\begin{aligned}
Y^{\prime}(x, \lambda) & =-\sqrt{\lambda} \sin (\sqrt{\lambda} x) I_{d}-\cos (\sqrt{\lambda} x) A+\int_{0}^{x} \cos (\sqrt{\lambda}(x-t)) P(t) Y(x, \lambda) d t \\
& =-\sqrt{\lambda} \sin (\sqrt{\lambda} x) I_{d}-\cos (\sqrt{\lambda} x) A+\frac{\cos (\sqrt{\lambda} x)}{2} \int_{0}^{x} P(x) d t+o(\exp |\operatorname{Im} \sqrt{\lambda}|) .
\end{aligned}
$$

Let

(10)

$$
\begin{aligned}
& \mathcal{F}_{P}(\lambda) \equiv B Y(1, \lambda)+Y^{\prime}(1, \lambda) \\
&=\sqrt{\lambda}\left\{-\sin (\sqrt{\lambda}) I_{d}+\frac{1}{\sqrt{\lambda}}\left[B-A+\frac{1}{2} \int_{0}^{1} P(t) d t\right]\right. \cos (\sqrt{\lambda}) \\
&\left.+o\left(\frac{\exp |\operatorname{Im} \sqrt{\lambda}|}{\sqrt{\lambda}}\right)\right\} .
\end{aligned}
$$

Set

$$
\mathcal{G}_{P}(\lambda)=-\sin (\sqrt{\lambda}) I_{d}+\frac{1}{\sqrt{\lambda}}\left[B-A+\frac{1}{2} \int_{0}^{1} P(t) d t\right] \cos (\sqrt{\lambda})+o\left(\frac{|\exp | \operatorname{Im} \sqrt{\lambda} \mid}{\sqrt{\lambda}}\right) .
$$

Then, for $\lambda \in \mathbb{R} \backslash\{0\}$, $\exp |\operatorname{Im} \sqrt{\lambda}|=1$, then $\lambda$ is an eigenvalue of (15) if and only if $\mathcal{G}_{P}(\lambda)$ is singular. By Rouche's Theorem, the zeros of $\operatorname{det} \mathcal{G}_{P}(\lambda)$ are asymptotic to the zeros of

$$
\mathcal{H}_{P}(\lambda)=\operatorname{det}\left\{-\sin (\sqrt{\lambda}) I_{d}+\frac{1}{\sqrt{\lambda}}\left[B-A+\frac{1}{2} \int_{0}^{1} P(t) d t\right] \cos (\sqrt{\lambda})\right\} .
$$

Denote $\left\{a_{1}, a_{2}, \ldots, a_{d}\right\}$ to be the set of eigenvalues of $B-A+\frac{1}{2} \int_{0}^{1} P(t) d t$ with $a_{1} \leq a_{2} \leq \cdots \leq a_{d}$. Then the zeros of $\mathcal{H}_{P}(\lambda)$ consist of the zeros of

$$
\sin (\sqrt{\lambda})=\frac{a_{j}}{\sqrt{\lambda}} \cos (\sqrt{\lambda}), j=1,2, \ldots, d .
$$

Hence

$$
\tan (\sqrt{\lambda})=\frac{a_{j}}{\sqrt{\lambda}}, j=1,2, \ldots, d
$$


For $\lambda>>1$, we may denote the $n^{\text {th }}$ zero of the above equation by $\sqrt{\lambda_{d m+j}}=$ $(m+1) \pi+\delta_{d, m}^{j}$, where $m=\left[\frac{n}{d}\right]$ and $j=1,2, \ldots, d$. Thus, for $m$ large enough, we have $\left|\delta_{d, m}^{j}\right|<<1$ and

$$
\tan \left((m+1) \pi+\delta_{d, m}^{j}\right)=\tan \left(\delta_{d, m}^{j}\right)=\frac{a_{j}}{(m+1) \pi+\delta_{d, m}^{j}} .
$$

Therefore

$$
\delta_{d, m}^{j}=\frac{a_{j}}{(m+1) \pi}+O\left(\frac{1}{m^{2}}\right) .
$$

We obtain

$$
\begin{aligned}
\sqrt{\lambda_{d m+j}} & =(m+1) \pi+\frac{a_{j}}{(m+1) \pi}+O\left(\frac{1}{m^{2}}\right) \\
& =(m+1) \pi+O\left(\frac{1}{m}\right) .
\end{aligned}
$$

This completes the proof.

From the above estimate of the eigenvalues, we obtain the asymptotics of the nodal points.

Theorem 2.3. Suppose $\mathbf{y}\left(x, \lambda_{n}\right)$ is a solution of the vectorial problem (2) and (5) of type (CZ) corresponding to the eigenvalue $\lambda_{n}$, and $\left\{x_{k}^{(n)}\right\}$ is the nodal set of $\mathbf{y}\left(x, \lambda_{n}\right)$. Then let $m=\left[\frac{n}{d}\right]$,

$$
x_{k}^{(n)}=\frac{k+\frac{1}{2}}{m+1}+O\left(\frac{1}{n^{2}}\right), \quad k=0,1,2, \ldots, m,
$$

for sufficiently large $n$.

Proof. Let $Y(x, \lambda)$ be the solution of (8), and let $\mathbf{v}_{n} \in \mathbb{R}^{d}-\{\mathbf{0}\}$ be a unit null vector of $\mathcal{F}_{P}\left(\lambda_{n}\right)$ such that $Y\left(x, \lambda_{n}\right) \mathbf{v}_{n}$ is an eigenfunction of (2) and (5) of type (CZ). Then by (9),

$$
Y\left(\frac{\left(k+\frac{1}{4}\right) \pi}{\sqrt{\lambda_{n}}}, \lambda_{n}\right) \mathbf{v}_{n}=\cos \left(\left(k+\frac{1}{4}\right) \pi\right) \mathbf{v}_{n}+O\left(\frac{1}{n}\right)
$$

and

$$
Y\left(\frac{\left(k+\frac{3}{4}\right) \pi}{\sqrt{\lambda_{n}}}, \lambda_{n}\right) \mathbf{v}_{n}=\cos \left(\left(k+\frac{3}{4}\right) \pi\right) \mathbf{v}_{n}+O\left(\frac{1}{n}\right) .
$$

Let $y_{n, j}(x)$ be the $j$-th component of $Y\left(x, \lambda_{n}\right) \mathbf{v}_{n}$. Since $\cos \left(\left(k+\frac{1}{4}\right) \pi\right) \cos \left(\left(k+\frac{3}{4}\right) \pi\right)<$ 0 , by the intermediate value theorem, there is some $x_{k, j}^{(n)}$ in $\left(\frac{\left(k+\frac{1}{4}\right) \pi}{\sqrt{\lambda_{n}}}, \frac{\left(k+\frac{3}{4}\right) \pi}{\sqrt{\lambda_{n}}}\right)$ such that $y_{n, j}\left(x_{k, j}^{(n)}\right)=0$. Then, by the (CZ) property, all these $x_{k, j}^{(n)}$ 's coincide at a point $x_{k}^{(n)}$, say. So $Y\left(x_{k}^{(n)}, \lambda_{n}\right) \mathbf{v}_{n}=\mathbf{0}$. Let $x_{k}^{(n)}=\frac{\left(k+\frac{1}{2}\right) \pi}{\sqrt{\lambda_{n}}}+C_{k}^{(n)}$, where $0 \leq\left|C_{k}^{(n)}\right| \leq$ $\frac{\pi}{4 \sqrt{\lambda_{n}}}$. Then

$$
\mathbf{0}=Y\left(x_{k}^{(n)}, \lambda_{n}\right) \mathbf{v}_{n}=\left[\cos \left(\left(k+\frac{1}{2}\right) \pi+\sqrt{\lambda_{n}} C_{k}^{(n)}\right)+O\left(\frac{1}{n}\right)\right] \mathbf{v}_{n} .
$$

Hence

$$
0=\cos \left(\left(k+\frac{1}{2}\right) \pi+\sqrt{\lambda_{n}} C_{k}^{(n)}\right)+O\left(\frac{1}{n}\right)-\sin \left(\sqrt{\lambda_{n}} C_{k}^{(n)}\right)+O\left(\frac{1}{n}\right) .
$$


We can obtain $C_{k}^{(n)}=O\left(\frac{1}{n^{2}}\right)$. Therefore,

$$
\begin{aligned}
x_{k}^{(n)} & =\frac{\left(k+\frac{1}{2}\right) \pi}{\sqrt{\lambda_{n}}}+C_{k}^{(n)} \\
& =\frac{k+\frac{1}{2}}{m+1}+O\left(\frac{1}{n^{2}}\right),
\end{aligned}
$$

where $k=0,1,2, \ldots, m$.

Corollary 2.4. With the same assumptions as in Theorem 2.3, we have

(a) $l_{k}^{(n)}=x_{k+1}^{(n)}-x_{k}^{(n)}=\frac{1}{n}+O\left(\frac{1}{n^{2}}\right)$,

(b) $\sqrt{\lambda_{n}}\left(x_{k+1}^{(n)}-x_{k}^{(n)}\right)=\pi+O\left(\frac{1}{n}\right)$,

(c) $\sqrt{\lambda_{n}} x_{k}^{(n)}=\left(k+\frac{1}{2}\right) \pi+O\left(\frac{1}{n}\right)$.

\section{Proof of the Main theorem}

The following lemma follows from [12, Lemma 2.4]. One can also prove it directly using compound angle formulae and the Riemann-Lebesgue Lemma.

Lemma 3.1. Suppose $Y\left(x, \lambda_{n}\right)$ is the solution of (8). Let $\mathbf{v}_{n}$ be a unit null vector of $\mathcal{F}_{P}\left(\lambda_{n}\right)$ (10) such that $Y\left(x, \lambda_{n}\right) \mathbf{v}_{n}$ is an eigenfunction of (2) and (5) corresponding to the eigenvalue $\lambda_{n}$ and is of type (CZ) with the nodal set $\left\{x_{k}^{(n)}\right\}$. Then as $n \rightarrow \infty$,

(a) for each $l, m$, there is an $\eta_{l m, k}^{(n)} \in\left[x_{k}^{(n)}, x_{k+1}^{(n)}\right]$ such that

$$
\begin{aligned}
\int_{x_{k}^{(n)}}^{x_{k+1}^{(n)}} & \sin \left(\sqrt{\lambda_{n}}\left(x_{k+1}^{(n)}-t\right)\right) P(t) Y\left(t, \lambda_{n}\right) d t \\
& =\frac{l_{k}^{(n)} \sin \left(\sqrt{\lambda_{n}} x_{k+1}^{(n)}\right)}{2}\left[p_{l m}\left(\eta_{l m, k}^{(n)}\right)\right]_{l, m=1}^{d}+O\left(\frac{1}{n^{2}}\right),
\end{aligned}
$$

(b)

$$
\int_{0}^{x_{k}^{(n)}}\left[\sin \left(\sqrt{\lambda_{n}}\left(x_{k+1}^{(n)}-t\right)\right)+\sin \left(\sqrt{\lambda_{n}}\left(x_{k}^{(n)}-t\right)\right)\right] P(t) Y\left(t, \lambda_{n}\right) d t=o\left(\frac{1}{n}\right) .
$$

Now we set out to prove our main theorem.

Proof of Theorem 1.4. We shall show that $P(x)$ is simultaneously diagonalizable in part(a), and $A$ and $B$ are diagonalizable in part(b).

Let $\left\{\lambda_{n_{j}}\right\}$ be the eigenvalues of (22) and (5) and $Y(x, \lambda)$ be the solution of (8). Then

$$
\mathcal{F}_{P}\left(\lambda_{n_{j}}\right) \equiv B Y\left(1, \lambda_{n_{j}}\right)+I_{2} Y^{\prime}\left(1, \lambda_{n_{j}}\right)
$$

is singular. Hence there are unit null vectors $\left\{\mathbf{v}_{n_{j}}\right\}$ of $\mathcal{F}_{P}\left(\lambda_{n_{j}}\right)$ so that $Y\left(x, \lambda_{n_{j}}\right) \mathbf{v}_{n_{j}}$ are eigenfunctions of (21) and (51) corresponding to $\lambda_{n_{j}}$. We may assume that $\mathbf{v}_{n_{j}}$ is convergent to some unit vector $\mathbf{v}$. Suppose $Y\left(x, \lambda_{n_{j}}\right) \mathbf{v}_{n_{j}}$ is an eigenfunction of type (CZ). Let $\left\{x_{k}^{\left(n_{j}\right)}: j=1,2, \ldots ; k=0,1,2, \ldots,\left[\frac{n_{j}}{2}\right]\right\}$ be the nodal set, which is dense in $(0,1)$. Then for fixed $x_{0} \in(0,1)$, there are intervals $\left(x_{k_{j}}^{\left(n_{j}\right)}, x_{k_{j}+1}^{\left(n_{j}\right)}\right)$ containing $x_{0}$, such that

$$
\lim _{j \rightarrow \infty} x_{k_{j}}^{\left(n_{j}\right)}=x_{0}=\lim _{j \rightarrow \infty} x_{k_{j}+1}^{\left(n_{j}\right)}
$$


Since

$$
Y\left(x_{k_{j}}^{\left(n_{j}\right)}, \lambda_{n_{j}}\right) \mathbf{v}_{n_{j}}=\mathbf{0}=Y\left(x_{k_{j}+1}^{\left(n_{j}\right)}, \lambda_{n_{j}}\right) \mathbf{v}_{n_{j}},
$$

we have, by Lemma 2.1

$$
\begin{aligned}
0= & Y\left(x_{k_{j}}^{\left(n_{j}\right)}, \lambda_{n_{j}}\right) \mathbf{v}_{n_{j}}+Y\left(x_{k_{j}+1}^{\left(n_{j}\right)}, \lambda_{n_{j}}\right) \mathbf{v}_{n_{j}}, \\
= & {\left[\cos \left(\sqrt{\lambda_{n_{j}}} x_{k_{j}}^{\left(n_{j}\right)}\right)+\cos \left(\sqrt{\lambda_{n_{j}}} x_{k_{j}+1}^{\left(n_{j}\right)}\right)\right] \mathbf{v}_{n_{j}} } \\
& -\frac{\sin \left(\sqrt{\lambda_{n_{j}}} x_{k_{j}}^{\left(n_{j}\right)}\right)+\sin \left(\sqrt{\lambda_{n_{j}}} x_{k_{j}+1}^{\left(n_{j}\right)}\right)}{\sqrt{\lambda_{n_{j}}}} A \mathbf{v}_{n_{j}} \\
& +\left[\int_{0}^{x_{k_{j}}^{\left(n_{j}\right)}} \frac{\sin \left(\sqrt{\lambda_{n_{j}}}\left(x_{k_{j}}^{\left(n_{j}\right)}-t\right)\right)+\sin \left(\sqrt{\lambda_{n_{j}}}\left(x_{k_{j}+1}^{\left(n_{j}\right)}-t\right)\right)}{\sqrt{\lambda_{n_{j}}}} P(t) Y\left(t, \lambda_{n_{j}}\right) d t\right] \mathbf{v}_{n_{j}} \\
& +\left[\int_{x_{k_{j}}^{\left(n_{j}\right)}}^{x_{k_{j}+1}^{\left(n_{j}\right)}} \frac{\sin \left(\sqrt{\lambda_{n_{j}}}\left(x_{k_{j}+1}^{\left(n_{j}\right)}-t\right)\right)}{\sqrt{\lambda_{n_{j}}}} P(t) Y\left(t, \lambda_{n_{j}}\right) d t\right] \mathbf{v}_{n_{j}} .
\end{aligned}
$$

By Lemma 3.1 there is $\eta_{l m, k_{j}}^{\left(n_{j}\right)} \in\left[x_{k_{j}}^{\left(n_{j}\right)}, x_{k_{j}+1}^{\left(n_{j}\right)}\right]$ such that

$$
\begin{aligned}
& \int_{x_{k_{j}}^{\left(n_{j}\right)}}^{x_{k_{j}+1}^{\left(n_{j}\right)}} \frac{\sin \left(\sqrt{\lambda_{n_{j}}}\left(x_{k_{j}+1}^{\left(n_{j}\right)}-t\right)\right)}{\sqrt{\lambda_{n_{j}}}} P(t) Y\left(t, \lambda_{n_{j}}\right) d t \\
& =\frac{l_{k_{j}}^{\left(n_{j}\right)} \sin \left(\sqrt{\lambda_{n_{j}}} x_{k_{j}+1}^{\left(n_{j}\right)}\right)}{2 \sqrt{\lambda_{n_{j}}}}\left[p_{l m}\left(\eta_{l m, k_{j}}^{\left(n_{j}\right)}\right]_{l, m=1}^{2}+O\left(\frac{1}{n_{j}^{3}}\right) .\right.
\end{aligned}
$$

Also, by Corollary 2.4,

$$
\begin{aligned}
& \sin \left(\sqrt{\lambda_{n_{j}}} x_{k_{j}}^{\left(n_{j}\right)}\right)+\sin \left(\sqrt{\lambda_{n_{j}}} x_{k_{j}+1}^{\left(n_{j}\right)}\right) \\
& =2 \sin \left(\sqrt{\lambda_{n_{j}}}\left(\frac{x_{k_{j}}^{\left(n_{j}\right)}+x_{k_{j}+1}^{\left(n_{j}\right)}}{2}\right)\right) \cos \left(\sqrt{\lambda_{n_{j}}}\left(\frac{x_{k_{j}}^{\left(n_{j}\right)}-x_{k_{j}+1}^{\left(n_{j}\right)}}{2}\right)\right) \\
& =O\left(\frac{1}{n_{j}^{2}}\right) .
\end{aligned}
$$

Hence by the above two equations and Lemma 3.1(b), we can rewrite (11) as

$$
\left[p_{l m}\left(\eta_{l m, k_{j}}^{\left(n_{j}\right)}\right)\right]_{l, m=1}^{2} \mathbf{v}_{n_{j}}=-\frac{2 \sqrt{\lambda_{n_{j}}}\left[\cos \left(\sqrt{\lambda_{n_{j}}} x_{k_{j}}^{\left(n_{j}\right)}\right)+\cos \left(\sqrt{\lambda_{n_{j}}} x_{k_{j}+1}^{\left(n_{j}\right)}\right)\right]}{l_{k_{j}}^{\left(n_{j}\right)} \sin \left(\sqrt{\lambda_{n_{j}}} x_{k_{j}+1}^{\left(n_{j}\right)}\right)} \mathbf{v}_{n_{j}}+o(1) .
$$

Since $P$ is a continuous matrix-valued function, $\left[p_{l m}\left(\eta_{l m, k_{j}}^{\left(n_{j}\right)}\right)\right]_{l, m=1}^{2}$ converges to $P\left(x_{0}\right)$, as $j \rightarrow \infty$. On the other hand, since $\left\|\mathbf{v}_{n_{j}}\right\|=1$ for all $n_{j}, \mathbf{v}_{n_{j}}$ converges to some $\mathbf{v}$. Therefore, taking the limit as $j \rightarrow \infty$, the left-hand side of the above equation converges to $P\left(x_{0}\right) \mathbf{v}$, and the corresponding right-hand side becomes

$$
\lim _{j \rightarrow \infty}-\frac{2 \sqrt{\lambda_{n_{j}}}\left[\cos \left(\sqrt{\lambda_{n_{j}}} x_{k_{j}}^{\left(n_{j}\right)}\right)+\cos \left(\sqrt{\lambda_{n_{j}}} x_{k_{j}+1}^{\left(n_{j}\right)}\right)\right]}{l_{k_{j}}^{\left(n_{j}\right)} \sin \left(\sqrt{\lambda_{n_{j}}} x_{k_{j}+1}^{\left(n_{j}\right)}\right)} \mathbf{v}_{n_{j}}=c\left(x_{0}\right) \mathbf{v} .
$$


Hence

$$
P\left(x_{0}\right) \mathbf{v}=c\left(x_{0}\right) \mathbf{v} .
$$

Since $x_{0}$ is arbitrary in $(0,1)$, we can choose $x_{1} \neq x_{0}$ in $(0,1)$ and use the above argument for $x_{1}$ such that $\mathbf{v}$ is also an eigenvector of $P\left(x_{1}\right)$. Then $P\left(x_{0}\right)$ and $P\left(x_{1}\right)$ have the same eigenvector $\mathbf{v}$. That is, $\mathbf{v}$ is chosen independently for $x$.

Furthermore, let $\mathbf{u}$ be a unit vector orthogonal to $\mathbf{v}$. Since $P(x)$ is diagonalizable for all $x \in[0,1], \mathbf{u}$ has to be an eigenvector for $P(x)$. Thus there is another scalar $\tilde{c}(x)$, depending on $x$, such that $P(x) \mathbf{u}=\tilde{c}(x) \mathbf{u}$. Therefore we conclude that $P$ is simultaneously diagonalizable. In fact, $Q=[\mathbf{v}, \mathbf{u}]$ and $Q^{*} P Q=\left[\begin{array}{cc}c(x) & 0 \\ 0 & \tilde{c}(x)\end{array}\right]$. The proof of part (a) is complete.

Since $Y\left(x, \lambda_{n_{j}}\right) \mathbf{v}_{n_{j}}$ is an eigenfunction, and

$$
\begin{aligned}
& \int_{0}^{x_{1}^{\left(n_{j}\right)}} \sin \left(\sqrt{\lambda_{n_{j}}}\left(x_{1}^{\left(n_{j}\right)}-t\right)\right) P(t) Y\left(t, \lambda_{n_{j}}\right) d t \\
& =\sin \left(\sqrt{\lambda_{n_{j}}} x_{1}^{\left(n_{j}\right)}\right) \int_{0}^{x_{1}^{\left(n_{j}\right)}} \cos \left(\sqrt{\lambda_{n_{j}}} t\right) P(t) Y\left(t, \lambda_{n_{j}}\right) d t \\
& \quad-\cos \left(\sqrt{\lambda_{n_{j}}} x_{1}^{\left(n_{j}\right)}\right) \int_{0}^{x_{1}^{\left(n_{j}\right)}} \sin \left(\sqrt{\lambda_{n_{j}}} t\right) P(t) Y\left(t, \lambda_{n_{j}}\right) d t \\
& =\frac{\sin \left(\sqrt{\lambda_{n_{j}}} x_{1}^{\left(n_{j}\right)}\right)}{2} \int_{0}^{x_{1}^{\left(n_{j}\right)}} P(t) d t+O\left(\frac{1}{n_{j}^{2}}\right) \\
& =O\left(\frac{1}{n_{j}}\right)
\end{aligned}
$$

we have

$$
\begin{aligned}
\mathbf{0} & =Y\left(x_{1}^{\left(n_{j}\right)}, \lambda_{n_{j}}\right) \mathbf{v}_{n_{j}} \\
& =\left[\cos \left(\sqrt{\lambda_{n_{j}}} x_{1}^{\left(n_{j}\right)}\right) I_{2}-\frac{\sin \left(\sqrt{\lambda_{n_{j}}} x_{1}^{\left(n_{j}\right)}\right)}{\sqrt{\lambda_{n_{j}}}} A+O\left(\frac{1}{n_{j}^{2}}\right)\right] \mathbf{v}_{n_{j}}
\end{aligned}
$$

Hence,

$$
A \mathbf{v}_{n_{j}}=\sqrt{\lambda_{n_{j}}} \cot \left(\sqrt{\lambda_{n_{j}}} x_{1}^{\left(n_{j}\right)}\right) \mathbf{v}_{n_{j}}+O\left(\frac{1}{n_{j}}\right)
$$

Let $j \rightarrow \infty$. Then $\mathbf{v}_{n_{j}}$ tends to the same vector $\mathbf{v}$, and so the sequence

$$
\sqrt{\lambda_{n_{j}}} \cot \left(\sqrt{\lambda_{n_{j}}} x_{1}^{\left(n_{j}\right)}\right)
$$

has a limit $C$ in $\mathbb{R}$. Thus, $A \mathbf{v}=C \mathbf{v}$. With a similar argument as in (a), we conclude that $\mathrm{A}$ is diagonalizable by the same unitary matrix $Q$. Similarly, if we consider the matrix differential equation

$$
\left\{\begin{array}{l}
-Y^{\prime \prime}+P(x) Y=\lambda I_{2} Y \\
Y(1, \lambda)=I_{2}, Y^{\prime}(1, \lambda)=-B
\end{array}\right.
$$

and analyses $Y\left(x_{n_{j-1}}^{\left(n_{j}\right)}, \lambda_{n_{j}}\right) \mathbf{v}_{n_{j}}$, then $B$ is also diagonalizable by $Q$. Hence $P(x), A$, and $B$ are simultaneously diagonalizable.

With the above proof, we have the following theorem for higher-dimensional vectorial systems. 
Corollary 3.2. Let $d>2$ and $\mathbf{v}_{n}$ be a unit null vector of $\mathcal{F}_{P}\left(\lambda_{n}\right)$ for each $n$. If $\mathbf{y}_{n}(x)=Y\left(x, \lambda_{n}\right) \mathbf{v}_{n}$ is of type (CZ) for each $n$ and $\operatorname{span}\left\{\mathbf{v}_{n}: n \in \mathbb{N}\right\}$ contains $d$ orthonormal vectors in $\mathbb{R}^{d}$, then the vectorial problem (21) and (5) is ( $\star$ )simultaneously diagonalizable.

\section{ACKNOWLEDGEMENTS}

The authors would like to thank Professor Hua-Huai Chern for helpful discussions. We are partially supported by the National Science Council, Taiwan, R.O.C.

\section{REFERENCES}

[1] Z.S. Agranovich and V.A. Marchenko, The Inverse Problem of Scattering Theory, Gordon and Breach, New York, 1963. MR0162497(28:5696)

[2] R. Carlson, Large eigenvalues and trace formulas for matrix Sturm-Liouville problems, SIAM J. Math. Anal. 30 (1999), No. 5, 949-962. MF1709782 (2000f:34167)

[3] O.H. Hald and J.R. McLaughlin, Solutions of inverse nodal problems, Inverse Problems 5 (1989), 307-347. MR0999065 (90c:34015)

[4] Y.T. Chen, Y.H. Cheng, C.K. Law and J. Tsay, $L^{1}$ convergence of the reconstruction formula for the potential function, Proc. Amer. Math. Soc. 130 (2002), no. 8, 2319-2324. MR1896415 $(2002 \mathrm{k}: 34021)$

[5] H.H. Chern, C.K. Law, and H.J. Wang, Extensions of Ambarzumyan's theorem to general boundary conditions, J. Math. Anal. Appl. 263 (2001), no. 2, 333-342. MR1866051 (2002g:34195)

[6] I.C. Gohberg and M.G. Krel̆n, Theory and Applications of Volterra Operators in Hilbert Space, Translations of Mathematical Monographs, Vol. 24, American Mathematical Society, Providence, 1970. MR0264447|(41:9041)

[7] C.K. Law, C.L. Shen and C.F. Yang, The inverse nodal problem on the smoothness of the potential function, Inverse Problems 15 (1999), 253-263; Errata, 17(2001), 361-364. MR.1675348(2000a:34020)

[8] J.R. McLaughlin, Inverse spectral theory using nodal points as data - a uniqueness result, $J$. Diff. Eqns. 73 (1988), 354-362. MR.0943946 (89f:34035)

[9] C.L. Shen, On the nodal sets of the eigenfunctions of the string equation, SIAM J. Math. Anal. 19 (1988), No. 6, 1419-1424. MR0965261 (89j:34035)

[10] C.L. Shen and C.T. Shieh, Two inverse eigenvalue problems for vectorial Sturm-Liouville equations, Inverse Problems 14 (1998), no. 5, 1331-1343. MR 1654647 (99j:34016)

[11] C.L. Shen and C.T. Shieh, On the multiplicity of eigenvalues of a vectorial Sturm-Liouville differential equation and some related spectral problems, Proc. Amer. Math. Soc. 127 (1999), no. 10, 2943-2952. MR1622977 (2000a:34165)

[12] C.L. Shen and C.T. Shieh, An inverse nodal problem for vectorial Sturm-Liouville equations, Inverse Problems 16 (2000), 349-356. MR1766766 (2001e:34024)

[13] X.F. Yang, A solution of the inverse nodal problem, Inverse Problems 13 (1997), 203-213. MR,1435878(98c:34017)

Department of Applied Mathematics, National Sun Yat-Sen University, Kaohsiung, TAiWAN 804, Republic of China

E-mail address: jengyh@math.nsysu.edu.tw

Department of Mathematics, Tamkang University, Tamsui, Taipei County, Tainan 251, Republic of China

E-mail address: ctshieh@math.tku.edu.tw

Department of Applied Mathematics, National Sun Yat-Sen University, Kaohsiung, TAiWAN 804, Republic of China

E-mail address: law@math.nsysu.edu.tw 